\section{Atenção à saúde bucal e a descentralização da saúde no Brasil: estudo de dois casos exemplares no Estado da Bahia}

\author{
Oral health care and health decentralization in \\ Brazil: two case studies in Bahia State
}

Sônia Cristina Lima Chaves 1

Lígia Maria Vieira-da-Silva ${ }^{2}$

\footnotetext{
${ }^{1}$ Faculdade de Odontologia, Universidade Federal da Bahia, Salvador, Brasil. 2 Instituto de Saúde Coletiva, Universidade Federal da Bahia, Salvador, Brasil.

Correspondência S. C. L. Chaves Faculdade de Odontologia, Universidade Federal da Bahia

Rua Araújo Pinho, 72, 6o andar, Salvador, $B A$ 40110-912, Brasil. schaves@ufba.br
}

\begin{abstract}
A comparative case study was performed in two selected municipalities in the State of Bahia, Brazil, to discuss the relationship between health system decentralization and characteristics of oral health services. A logical model was developed and submitted to an expert panel. Data were gathered through in-depth interviews, field observation, and analysis of secondary data from the National Health System database. The results point to the influence of municipal government characteristics on oral health practice. One municipality was classified as having an intermediate standard of oral health system implementation (50\%), while the other showed only $11 \%$ implementation. The study showed that the decentralization process has not been accompanied by an effort to improve management capacity in the local oral health services, despite the transfer offunds to this area.
\end{abstract}

Oral Health; Health Evaluation; Health Policy

\section{Introdução}

O processo de descentralização das ações de saúde no Brasil, iniciado na década de 80, tem-se caracterizado por uma redefinição das funções e competências entre os níveis federal, estadual e municipal, com ênfase na municipalização das ações de saúde, conforme previsto nas Normas Operacionais Básicas (NOB) 93 e 96 1. Em algumas situações, esse processo foi acompanhado pela definição e implementação de estratégias desencadeadoras de mudanças nos modelos de atenção à saúde 2 voltados para a promoção da saúde, organização da oferta e territorialização 3 . Posteriormente, a Norma Operacional de Assistência à Saúde (NOAS 01/2001) significou uma tentativa de reorientação da descentralização a fim de também considerar a regionalização como estratégia de gestão 4 .

No curso do processo de consolidação do Sistema Único de Saúde (SUS), têm-se verificado esforços voltados para a reorganização da saúde bucal na atenção básica no nível municipal, em geral e particularmente, por intermédio do Programa de Agentes Comunitários de Saúde (PACS) e do Programa Saúde da Família (PSF) 5,6,7,8. A Política Nacional de Saúde Bucal, a partir de 2003, tem apoiado essas iniciativas pelo aumento substancial de investimentos para o setor odontológico ${ }^{9}$. Contudo, não está clara a "imagem-objetivo" da atenção à saúde bucal a ser implementada nos municípios brasileiros, 
ainda que alguns documentos apontem para certas prioridades no âmbito do PSF e abertura de centros odontológicos especializados para a média complexidade (endodontia e prótese) 9,10,11.

Há poucos estudos sobre como se desenvolvem as práticas de saúde bucal nos serviços odontológicos públicos no Brasil 12,13,14. Os trabalhos de Serra 15, Roncalli 12 e Peres 13, desenvolvidos nos anos 90, investigaram algumas intervenções municipais exitosas de reorganização das ações odontológicas no setor público em Curitiba (Paraná), Campinas (São Paulo), Belo Horizonte e Ipatinga (Minas Gerais). Nesses casos, o principal fator relacionado ao êxito tem sido o apoio do governo local 12,15. Esses estudos apontam para a importância da investigação também dos componentes da gestão municipal mais favoráveis para o desenvolvimento da atenção à saúde bucal pública do país 12,13,15.

Além disso, trabalho anterior, de síntese da literatura sobre a efetividade das estratégias para o controle e prevenção da cárie, apontou resultados discordantes entre estudos que testavam métodos semelhantes, reforçando a necessidade de serem investigados os diferentes contextos de implantação das intervenções preventivas em saúde bucal 16. Assim, o presente trabalho buscou analisar em que medida a descentralização da gestão da atenção à saúde tem influenciado a (re)organização dos serviços odontológicos em municípios da Bahia, na perspectiva da consolidação de práticas organizacionais, clínicas, preventivas e coletivas no nível local, articuladas aos princípios do SUS 10,11. Com essa finalidade, esse estudo estimou o grau de implantação dos componentes relacionados à organização da atenção à saúde bucal em municípios selecionados do Estado da Bahia. Também formulou uma imagem-objetivo da atenção à saúde bucal em municípios brasileiros com base no consenso de um comitê de experts especialmente constituído para este fim. Além disso, a investigação buscou compreender que fatores podem estar contribuindo ou obstando o processo de reorganização da atenção à saúde bucal no setor público.

\section{Metodologia}

\section{Desenho do estudo e seleção dos casos}

A análise da implantação 17 dos processos relacionados com a reorganização da atenção à saúde bucal foi realizada por meio de estudo de dois casos exemplares de municípios do Estado da Bahia. Os municípios selecionados, com população aproximada superior a 100 mil habitantes e habilitados na gestão plena do sistema muni- cipal de saúde, segundo a NOB 9618 foram classificados em diferentes graus de implantação da gestão descentralizada do SUS em estudo anterior 19 (Tabela 1). Esses municípios foram selecionados dentre cinco outros considerados como casos exemplares da descentralização da gestão da saúde na Bahia. A razão da escolha decorreu do fato de os mesmos se encontrarem em estágios extremos em relação ao grau de implantação da descentralização, ou seja, um deles foi classificado como apresentando grau avançado de implantação da gestão descentralizada do SUS (município C) e o outro foi considerado como incipiente (município E) 19.

\section{Modelo teórico-lógico e a imagem-objetivo}

Foi elaborado um "modelo lógico" 20 que relaciona a reorganização da atenção à saúde bucal tanto às características do governo local quanto àquelas da gestão e dos agentes das práticas. Um governo municipal que possua um projeto de reorganização das ações de saúde desenvolvido por profissionais capacitados e com apoio político e popular para implementá-lo cria condições favoráveis ao desenvolvimento e operacionalização de mudanças também nas políticas específicas como é o caso daquela voltada para a saúde bucal (Figura 1). Essas características do governo propostas por Matus 21, foram analisadas para cinco municípios do Estado da Bahia na primeira etapa da pesquisa ${ }^{19}$. No presente artigo, foram comparadas as características da gestão e das práticas específicas de atenção à saúde bucal em dois municípios que diferiam em relação às características de governo e de gestão global da saúde.

A existência de um projeto de governo voltado para a mudança do modelo assistencial constitui-se em espaço favorável à formulação de políticas de saúde bucal que priorizem o controle de riscos e causas. Por sua vez, as práticas orientadas por tal política deverão incluir ações educativas em grupos priorizados e práticas clínicas visando à autonomia. Por outro lado, uma gestão que se apóie no planejamento estratégico poderá dimensionar melhor os recursos ampliando acesso e cobertura. Essas ações de reorganização da atenção à saúde bucal poderão ter impacto na redução das cáries e de outros problemas identificados.

\section{Consenso entre experts sobre a imagem- objetivo da atenção a saúde bucal proposta}

Foi elaborada uma matriz contendo as dimensões e critérios para avaliação, derivada do modelo teórico-lógico (Tabela 2), que foi enviada a 
Dados sócio-demográficos, sanitários, políticos e do sistema de saúde nos municípios C e E, Bahia, Brasil, 2003.

\begin{tabular}{|c|c|c|}
\hline Indicadores & Município C & Município E \\
\hline População aproximada * & 260.000 & 110.000 \\
\hline Urbanização (habitação em zona urbana) (\%) ** & 86,0 & 83,0 \\
\hline Extensão territorial $\left(\mathrm{km}^{2}\right)$ & 3.204 & 2.408 \\
\hline População alfabetizada (\%) ** & 65,7 & 57,7 \\
\hline População com renda insuficiente (\%) ** & 59,5 & 70,2 \\
\hline Domicílio com canalização de água rede geral (\%) ** & 78,8 & 53,0 \\
\hline Domicílio sem instalação sanitária (\%) ** & 11,1 & 14,7 \\
\hline 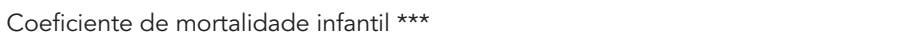 & 33,6 & 41,8 \\
\hline Mortalidade infantil proporcional por causas mal definidas em 1999 & 11,7 & 28,0 \\
\hline Gasto público com saúde per capita em U\$ (ano 2000) \# & 50,5 & 66,3 \\
\hline Gastos do Tesouro Municipal em saúde (\%) \#\# & 6,9 & 10,7 \\
\hline \multirow[t]{2}{*}{ Transferências federais para atenção básica per capita em R\$ e (U\$) em 2002 \#\#\# } & $\mathrm{R} \$ 24,2$ & $\mathrm{R} \$ 33,3$ \\
\hline & $(\cup \$ 12,1)$ & $(\cup \$ 16,7)$ \\
\hline Transferências federais para ações estratégicas per capita em $\mathrm{R} \$$ em 2002 \#\#\# & 6,7 & 0,24 \\
\hline Cobertura Programa de Agentes Comunitários de Saúde (\%) \#\# & 65,4 & 27,9 \\
\hline Cobertura do Programa Saúde da Família (\%) **,\#\#\# & 24,3 & 13,9 \\
\hline Gestão com continuidade administrativa & $\operatorname{Sim}$ & $\operatorname{Sim}$ \\
\hline
\end{tabular}

* Os dados de população foram aproximados visando à não-identificação dos municípios selecionados;

** Instituto Brasileiro de Geografia e Estatística 30;

*** Coeficiente de mortalidade infantil $=$ mortes infantis/mil nascidos vivos menores de um ano;

\# Gasto público per capita com saúde = total gastos/população total daquele ano;

\#\# Porcentagem de gastos do tesouro com saúde = total gastos municipais/receita total do município X 100. Em 2000, no município E, o Tesouro Municipal gastou $\mathrm{R} \$ 2.047 .910,25$ em saúde, o gasto federal foi de $\mathrm{R} \$ 10.017 .587,49$. No município C, o gasto total em saúde $\mathrm{R} \$ 26.546 .737,83$, sendo $\mathrm{R} \$ 2.547 .307,52$ do Tesouro Municipal e R\$23.999.430,31 do Tesouro Federal.

\#\#\# Fontes: Departamento de Informação e Informática do SUS 31,32.

um comitê de experts, com vistas à identificação acerca dos aspectos de maior consenso sobre uma possível "imagem-objetivo" da atenção à saúde bucal. O referido comitê foi formado por quatro especialistas em saúde bucal, de diferentes partes do país: três deles professores da área da odontologia social com doutorado e um quarto com mestrado e com experiência na gestão de serviços (Tabela 2). O método utilizado para o consenso foi o método Delphi 22,23. Cada membro respondia sobre sua concordância com a inclusão da referida dimensão proposta e atribuía uma nota de 0 a 10 em cada critério, em que 0 (zero) significa exclusão e 10 (dez), prioridade máxima.

Com base no modelo lógico elaborado 19 foram selecionados dois níveis de análise: (i) gestão da atenção à saúde bucal (organização do serviço); (ii) práticas desenvolvidas pelos profissionais na atenção básica. Para avaliar a "gestão da atenção à saúde bucal”, foram selecionadas as seguintes dimensões: (a) planejamento e programação, em que se busca identificar ações voltadas para o diagnóstico epidemiológico em saúde bucal, a programação e a avaliação; (b) suporte da gestão aos profissionais e às práticas na atenção básica, visa observar as ações do nível central no que se refere à capacitação, supervisão e suporte técnico e material às atividades dos mesmos; (c) oferta da assistência odontológica (cobertura potencial refere-se à cobertura potencial quanto à relação dentista/habitante no município; (d) utilização de serviços (cobertura real) segundo o Sistema de Informação Ambulatoriais (SIA-SUS) - referese ao número médio de procedimentos básicos produzidos pelo município em odontologia por pessoa; cobertura da primeira consulta odontológica - foi estimada considerando-se como padrão aquele proposto na Portaria no. 1.101/GM de 12 de junho de 2002 (Tabela 2) 24. Foi também considerada a concentração dos procedimentos coletivos na população de 0-14 anos e a razão restauração/extração; (e) integralidade da atenção em saúde bucal relaciona-se à oferta pelo município de ações especializadas em odontologia (endodontia, radiologia e prótese). Para o cálculo da cobertura potencial, decidiu-se trabalhar com uma relação de um dentista para cada 3 mil 
ESPAÇO GERAL - Contexto nacional

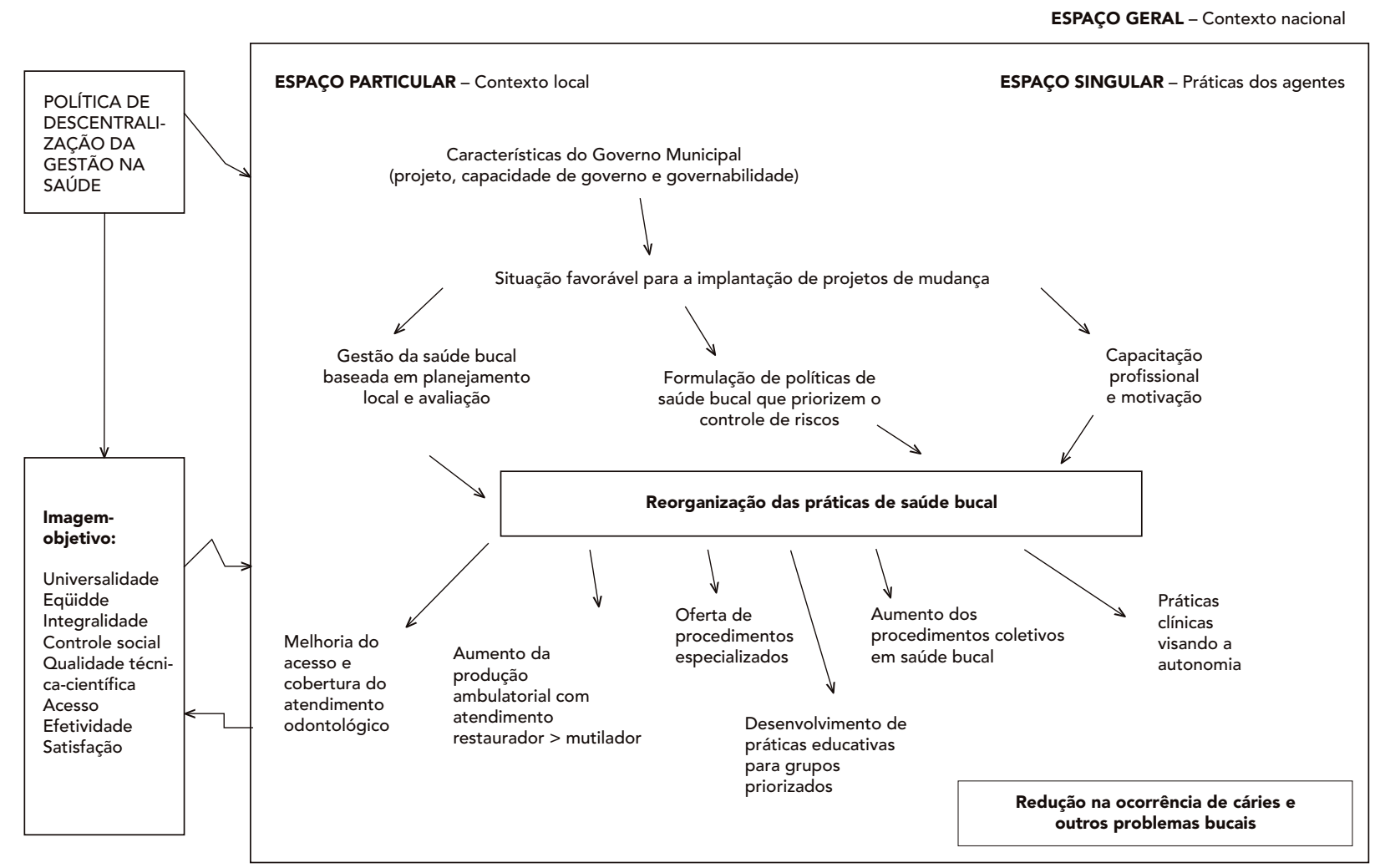

Adaptado de Vieira-da-Silva et al. ${ }^{19}$.

habitantes com renda insuficiente, próximo ao número de 3.450 indivíduos por equipe de saúde da família proposta pelo Ministério da Saúde 7. O uso da população com renda insuficiente como denominador decorreu do fato de se considerar essa proporção da população aquela que utilizaria potencialmente os serviços públicos de saúde no município.

Na análise do nível referente às práticas de saúde bucal, buscou-se verificar: (a) deslocamento do eixo sobre os danos para uma ação sobre os riscos - refere-se às estratégias para priorização de grupos a partir da identificação de riscos; (b) práticas educativas desenvolvidas pelos profissionais - corresponde às atividades de educação em saúde nas Unidades Básicas de Saúde (UBS) como salas-de-espera, grupos de escolares ou outros priorizados; (c) atividades junto aos Agentes
Comunitários de Saúde (ACS) - referiu-se a ações de capacitação e supervisão das ações de saúde bucal realizadas pelos mesmos; (d) práticas clínicas - observou-se se esta visava à autonomia dos pacientes no que se refere à manutenção da sua própria saúde bucal; (e) práticas desenvolvidas na família - correspondia às práticas de visitas domiciliares em saúde bucal e definição dos critérios para as mesmas; (f) acolhimento e vínculo - buscou-se identificar o respeito ao paciente e se este era prontamente atendido, sem filas, além de receber toda a informação necessária. Além disso, observou-se o estabelecimento de vínculo entre o profissional e o paciente e vice-versa (o paciente reconhece o dentista como seu dentista e este reconhece o paciente como seu); (g) atenção integral - observou-se o cuidado que o paciente recebia, desde ações de promoção, pre- 
Matriz de análise de implantação da atenção à saúde bucal em municípios brasileiros com a pontuação média dada pelo comitê de experts e respectivo desvio padrão, 2003

Nível 1 - Gestão da atenção à saúde bucal (organização do serviço) $(4,2$ pts.)
$\begin{aligned} & \text { Dimensão } \\ & \text { (pontuação máxima) }\end{aligned}$

Planejamento e programação (1,5 pt.)

\section{Diagnóstico epidemiológico}

a) Há "levantamentos" epidemiológicos periódicos realizados e este(s) têm sido utilizado(s) para o planejamento e definição de metas e ações (CPO-D, ceo-d e índice periodontal nas idades índices indicadas pela Organização Mundial da Saúde, por exemplo) (0,2 pt.);

b) Há incorporação do "levantamento" epidemiológico periódico pelas equipes de saúde bucal na atenção básica para o conhecimento e intervenção sobre a realidade local (0,2 pt.);

c) São realizadas avaliações das ações para verificar seu impacto epidemiológico (0,2pt.).

\section{Programação}

a) Planejamento é estratégico e envolve a consulta a diversos atores (equipe de saúde e usuários) (0,5 pt.);

b) O planejamento existe, porém é normativo (0,2 pt.).

\section{Avaliação}

a) Há avaliação sistemática do planejamento realizado, com análise de indicadores de produção, cobertura e efeito das ações (0,4 pt.);

b) A avaliação é feita a partir da análise de indicadores de produção dos serviços $(0,2$ pt.).

Suporte da gestão aos profissionais e às práticas na atenção básica $(0,5$ pt.)

Oferta da assistência odontológica (cobertura potencial) $(0,5$ pt.)

Utilização de serviços (cobertura real) segundo o Sistema de Informações Ambulatoriais (SIA-SUS) (0,7 pt.)

Integralidade da atenção em saúde bucal (1,0 pt.) interesse para o trabalho sem vínculo com a remuneração $(0,2$ pt.);

b) Há supervisão do trabalho desenvolvido, com suporte técnico e não apenas com controle sobre os trabalhadores $(0,1 \mathrm{pt}$.);

c) Há suporte material e instrumental para desenvolvimento das atividades $(0,2$ pt.).

a) A relação dentista no setor público/habitante/ano na última gestão deve ser igual ou superior ao padrão 1 dentista público para 3 mil habitantes com renda per capita inferior a $50 \%$ do salário mínimo (considerada população com renda insuficiente segundo o Atlas de Desenvolvimento Humano do Brasil 33) (0,3 pt.);

b) Houve ampliação da oferta de serviços odontológicos nos últimos quatro anos no município (0,2 pt.).

a) Os procedimentos ambulatoriais básicos habitante/ano atingem o padrão mínimo proposto pela Portaria no. 1.101/GM 24 (0,4 a 1,6 habitantes/ano) (0,1 pt.);

b) Concentração de consultas odontológicas habitante/ano na série histórica analisada (1998 a 2003) atinge o padrão de 0,25 consultas odontológicas habitantes/ano (equivale a 63\% do parâmetro mínimo de 0,4 procedimentos ambulatoriais segundo Portaria no. 1.101/GM 24) (0,2 pt.);

c) Os procedimentos coletivos habitante/ano atingem o padrão mínimo de quatro procedimentos coletivos habitantes/ano na população entre 0 e 14 anos (0,2 pt.);

d) Os procedimentos restauradores são em maior quantidade que os procedimentos cirúrgicos (a razão restauração/extração maior que 1) (0,2 pt.).

a) Há um sistema de referência das unidades básicas para a média complexidade (endodontia) (0,2 pt.);

b) Há um sistema de referência das unidades básicas para serviços de complementação diagnóstica (radiologia periapical) (0,2 pt.);

c) Há serviço de referência para prótese parcial removível e total (0,3 pt.);

d) Os procedimentos especializados em odontologia atingem o padrão proposto pela Portaria no. 1.101/GM 24 (0,04 a 0,06 procedimentos especializados/habitantes/ano) (0,3 pt.). a) O nível central promove oficinas regulares de capacitação dos profissionais sobre temáticas de

$\begin{array}{cc}\text { Pontuação } & \text { Nota } \\ \text { máxima } & \text { média (DP) }\end{array}$

0,6 $9,5( \pm 1,0)$

(continua) 
Tabela 2 (continuação)

\begin{tabular}{|c|c|c|c|}
\hline \multicolumn{4}{|c|}{ Nível 2 - Práticas desenvolvidas pelos profissionais atenção básica $(5,8$ pts.) } \\
\hline $\begin{array}{l}\text { Dimensão } \\
\text { (pontuação máxima) }\end{array}$ & Critérios/Padrões (pontuação máxima) & $\begin{array}{l}\text { Pontuação } \\
\text { máxima }\end{array}$ & $\begin{array}{l}\text { Nota } \\
\text { média (DP) }\end{array}$ \\
\hline $\begin{array}{l}\text { Deslocamento do eixo } \\
\text { sobre os danos para } \\
\text { uma ação sobre os riscos } \\
(1,0 \text { pt.) }\end{array}$ & $\begin{array}{l}\text { a) Há priorização de grupos de risco a partir de indicadores sócio-econômicos (0,6 pt.); } \\
\text { b) São desenvolvidas ações preventivo-educativas junto a grupos específicos priorizados }(0,4 \text { pt.). }\end{array}$ & 1,0 & $9,0( \pm 0,9)$ \\
\hline $\begin{array}{l}\text { Práticas educativas } \\
\text { desenvolvidas pelos } \\
\text { profissionais }(0,9 \text { pt.) }\end{array}$ & $\begin{array}{l}\text { a) A temática da saúde bucal está inserida nos grupos educativos ou salas-de-espera (0,5 pt.); } \\
\text { b) Existem ações preventivas regulares junto a escolares }(0,4 \text { pt.); } \\
\text { c) Existem ações educativas esporádicas, junto a indivíduos ou grupos }(0,3 \text { pt.). }\end{array}$ & 0,9 & $8,5( \pm 0,6)$ \\
\hline $\begin{array}{l}\text { Atividades junto aos } \\
\text { Agentes Comunitários de } \\
\text { Saúde (ACS) (0,8 pt.) }\end{array}$ & $\begin{array}{l}\text { a) Os ACS foram capacitados e treinados pelo profissional para atuação junto às micro-áreas } \\
(0,3 \text { pt.); } \\
\text { b) Há supervisão periódica do dentista sobre as ações de saúde bucal desenvolvidas pelos ACS } \\
\text { (0,2 pt.); } \\
\text { c) Há incorporação do ponto de vista dos ACS e de outros membros da equipe no planejamento de } \\
\text { atividades educativas e restauradoras do dentista ( } 0,3 \text { pt.). }\end{array}$ & 0,8 & $8,3( \pm 1,0)$ \\
\hline Práticas clínicas $(0,5$ pt.) & $\begin{array}{l}\text { a) Há higiene bucal supervisionada e uso de métodos preventivos antes ou após os procedimentos } \\
\text { restauradores ou cirúrgicos sendo que os mesmos estão embasados em um diagnóstico de risco/ } \\
\text { atividade de doença }(0,3 \text { pt.); } \\
\text { b) Há orientação profissional sistemática sobre os métodos preventivos nos momentos da consulta } \\
\text { odontológica no atendimento clínico }(0,2 \text { pt.); } \\
\text { c) Há prática restauradora tradicional, sem observar aspectos relacionados ao risco e a orientação ao } \\
\text { paciente }(0,2 \text { pt.). }\end{array}$ & 0,5 & $7,8( \pm 1,9)$ \\
\hline $\begin{array}{l}\text { Práticas desenvolvidas na } \\
\text { família ( } 0,8 \text { pt.) }\end{array}$ & $\begin{array}{l}\text { a) As visitas domiciliares são realizadas a partir de critérios de risco comuns da equipe (como } \\
\text { controle de diabéticos e gestantes) ou às doenças bucais como crianças pré-escolares, gestantes ou } \\
\text { adolescentes como reforço das práticas de auto-cuidado }(0,8 \text { pt.); } \\
\text { b) As visitas domiciliares são realizadas com a finalidade de atender indivíduos com impossibilidade } \\
\text { de deslocamento ou outros critérios }(0,4 \text { pt.). }\end{array}$ & 0,8 & $8,5( \pm 0,6)$ \\
\hline $\begin{array}{l}\text { Acolhimento e vínculo } \\
(1,0 \text { pt.) }\end{array}$ & $\begin{array}{l}\text { a) Há um respeito ao paciente e este é prontamente atendido, sem filas, e recebe toda a informação } \\
\text { necessária ao perguntar ou mesmo sem perguntar }(0,5 \text { pt.); } \\
\text { b) Observa-se que há o estabelecimento de um vínculo entre o profissional e o paciente e vice-versa } \\
\text { (o paciente reconhece o dentista como seu dentista e este reconhece o paciente como seu) } \\
\text { (0,5 pt.). }\end{array}$ & 1,0 & $9,5( \pm 0,6)$ \\
\hline $\begin{array}{l}\text { Atenção integral } \\
(0,8 \text { pt.) }\end{array}$ & $\begin{array}{l}\text { a) O paciente recebe no decorrer da assistência, ações de promoção, prevenção e recuperação de } \\
\text { sua saúde bucal }(0,4 \text { pt.); } \\
\text { b) Ao entrar em tratamento na Unidade Básica de Saúde, é garantido o término do mesmo } \\
\text { (0,4 pt.). }\end{array}$ & 0,8 & $8,8( \pm 1,5)$ \\
\hline
\end{tabular}

venção e recuperação de sua saúde bucal e se, além disso, ao iniciar o tratamento na UBS, era garantido o término do mesmo.

O somatório da pontuação máxima possível a ser atribuída para os dois níveis de análise foi de 10 pontos, sendo 4,2 pontos para o nível 1 (gestão e organização do serviço) e 5,8 pontos para o nível 2 (práticas de saúde bucal desenvolvidas) (Tabela 2).

\section{Técnicas de coleta e fonte de dados}

Para a análise de algumas das dimensões da matriz, as características e composição da produção ambulatorial odontológica foi descrita por intermédio de dados disponíveis no SIA-SUS 25, entre 1998 e 2003. Foi analisada a série histórica desses indicadores comparados com os padrões propostos pelo Ministério da Saúde 24 que estão descritos no interior da matriz de análise de im- 
plantação (Tabela 2). Os relatórios de gestão e planos de saúde dos referidos municípios, bem como os dados referentes à caracterização sócioeconômica e sanitária dos mesmos também foram consultados para análise do governo local.

\section{Características das práticas}

As características das práticas foram investigadas principalmente pelas técnicas qualitativas como entrevistas em profundidade e observação. As entrevistas ocorreram em dois momentos: no segundo semestre de 2001 (fase exploratória) e no segundo semestre de 2003.

Em cada município foram selecionadas para observação das práticas de saúde bucal e realização de entrevistas uma ou mais unidades consideradas de desempenho em saúde bucal "satisfatório" e outras cujo desempenho tivesse sido avaliado como "não satisfatório", por técnicos do nível central da Secretaria Municipal de Saúde respectiva. Foram indicadas Unidades de Saúde da Família (USF) para observação e trabalho de campo, embora as unidades tradicionais também pudessem ter sido indicadas.

Foram realizadas no município $\mathrm{C}$, entrevistas semi-estruturadas com o coordenador de saúde bucal, quatro cirurgiões-dentistas inseridos na atenção básica, quatro agentes comunitários de saúde e oito usuários do sistema público, num total de 17 entrevistas. No município E, foram entrevistados o coordenador de saúde bucal, três cirurgiões-dentistas, dois agentes comunitários de saúde e oito usuários do sistema, totalizando 14 entrevistas. Apenas um pesquisador conduziu todas as entrevistas. Foi obtido o consentimento por escrito junto aos profissionais e usuários participantes da investigação.

Todas as entrevistas foram gravadas e transcritas. As unidades de texto referentes a cada uma das dimensões derivadas do modelo lógico, ao lado de outras que emergiram durante o trabalho de campo foram analisadas no seu conjunto 26 . Utilizou-se o programa computacional para análise de dados não estruturados NUD*IST (QSR International Pty Ltd., Melbourne, Austrália).

\section{Classificação do grau de implantação da atenção à saúde bucal}

Cada nível e dimensões correspondentes, à luz das evidências obtidas, foram classificados quanto ao grau de implantação da atenção à saúde bucal em:

- Incipiente: se atingiu uma pontuação inferior a 33,3\% da pontuação máxima proposta;

- Intermediário: superior ou igual a 33,3\% e inferior a 66,6\% da pontuação máxima proposta;
- Avançado: se a pontuação foi superior ou igual a $66,6 \%$ da pontuação máxima proposta.

\section{Resultados}

O comitê de experts e a "imagem-objetivo"

Verificou-se elevado grau de consenso entre os experts consultados no que se refere à maioria dos critérios selecionados (Tabela 2). Houve maior discordância em relação aos indicadores de cobertura e oferta obtidos por intermédio do SIA-SUS, em relação aos quais as menores médias (entre 6,8 e 7) e os maiores desvios-padrão (entre 1,6 e 2,6) foram verificados. Além disso, no que se refere à cobertura potencial (dentista/habitante/ano), a polêmica esteve em considerar se, de fato, deveria haver uma cobertura ideal e inespecífica da prática odontológica, sem considerar o perfil epidemiológico da população sob análise. Apesar da baixa pontuação em relação a esses critérios, decidiu-se mantê-los tendo em vista que a despeito de sua inespecificidade e baixa sensibilidade quanto a aferir as necessidades de saúde bucal populacionais, o seu cálculo constitui-se numa aproximação da oferta de serviços podendo auxiliar na comparação entre municípios.

Já a pontuação dada às dimensões referentes à gestão foi muito próxima daquela dada às práticas assistenciais, o que faz supor que os especialistas consideram igualmente relevantes os dois níveis de atenção à saúde bucal.

\section{Algumas características sócio-demográficas e políticas dos municípios}

Ambos os municípios estudados estavam na gestão plena do sistema de saúde, possuíam porte populacional equivalente, continuidade político-administrativa nas duas últimas gestões, ou seja, havia razoável estabilidade administrativa para o desenvolvimento de ações (Tabela 1). Os indicadores relacionados às condições de vida apresentavam melhores escores no município C quando comparados ao município E (Tabela 1). Em contrapartida, o gasto público, per capita, em saúde, em 2000, foi maior no município E que no município C. Também a cobertura, tanto do PACS quanto do PSF, apresentou valores superiores no município $\mathrm{C}$ em relação ao município $\mathrm{E}$.

\section{Grau de implantação da atenção \\ à saúde bucal}

A atenção à saúde bucal no município $\mathrm{C}$ foi classificada como em estágio intermediário (50\%), 
enquanto no município E, ela se mostrou muito incipiente (11\%) (Tabela 3, Figura 2). No município $\mathrm{C}$, a maioria das dimensões foi classificada como intermediária com uma variação entre incipiente no que diz respeito ao acolhimento e planejamento (30 e 33,3\% de implantação, respectivamente) e elevada na dimensão da oferta de serviços e práticas educativas desenvolvidas (80 e 66,7\%, respectivamente) (Tabela 3, Figura 2). Por outro lado, o município E mostrou-se incipiente em todas as dimensões analisadas. Chama a atenção no município E a completa ausência de um sistema de referência para a média complexidade em saúde bucal (integralidade da atenção), e esse serviço era considerado obrigatório para municípios já habilitados na gestão plena do sistema de saúde, segundo a NOAS$2001^{4}$.

A relação dentista/habitante com renda insuficiente, em 1998, no município E foi de 1/12.313. Em 2003, esse indicador foi de um dentista para 8.931 habitantes com renda insuficiente. Contudo, essa "melhoria" pode ainda ser considerada insuficiente quando considerado o padrão de 1/3.000 habitantes, fato que corresponderia a uma cobertura potencial de $37,3 \%$. Por outro lado, no município $\mathrm{C}$, a cobertura potencial evoluiu de 37,8\% em 1998 (1 dentista/7.924 habitantes) para 96,9\% em 2003 (1 dentista/3.133 habitantes), praticamente atingindo o padrão proposto (Tabela 2). Cabe destacar que, apesar do aumento na oferta de serviços odontológicos no município $\mathrm{C}$, isso não se reflete nos indicadores de produção de serviços ambulatoriais no SIASUS na mesma proporção. Esta cobertura real se manteve intermediária (57,2\% de implantação), o que pode estar significando um baixo uso da capacidade instalada. Por outro lado, pode estar correspondendo a uma maior ênfase nas atividades coletivas em detrimento das atividades ambulatoriais (restaurações, extrações), merecendo análises posteriores (Tabela 3, Figura 2).

No que se refere ao planejamento das ações, nunca foi feito um inquérito epidemiológico em saúde bucal no município E. Houve uma iniciativa que ficou inconclusa, pois os dados, apesar de terem sido coletados, não foram tabulados, analisados e se "perderam" na Secretaria Municipal de Saúde, segundo relato da atual coordenadora do setor. Também no município C, foi feito apenas um inquérito epidemiológico de cárie dental em 2000, em áreas cobertas com o PSF. Este foi tabulado, analisado e apresentado ao grupo de profissionais para discussão de estratégias de intervenção, avaliação e acompanhamento contínuo das ações, o que deveria ser feito a cada três anos. Os profissionais referem alguns pontos positivos do inquérito, como a possibilidade de usar os dados epidemiológicos para analisar a realidade de sua área mediante uma comparação entre a situação local e as metas propostas pela Organização Mundial da Saúde e outros estudos nacionais.

Observou-se que a avaliação sistemática pela análise do inquérito epidemiológico ou pela análise das séries históricas de produção dos serviços por unidade de saúde não foi implementada.

O uso do instrumental epidemiológico, como componente de análise da situação de saúde, ainda que incipiente, voltado para escolares e restrito à cárie dental, necessita maior preparo das equipes ou a incorporação de profissionais especializados em gestão de sistemas de saúde ou epidemiologia para sua implementação, especialmente nas fases de tabulação, consolidação e análise dos dados. Tanto no município C quanto no município E, esta foi uma lacuna, embora com maior magnitude no último caso.

A programação e avaliação se constituíram em importantes problemas em ambos os municípios, ainda que mais grave no município $\mathrm{E}$, onde reuniões periódicas e sistemáticas não foram realizadas entre os profissionais e a coordenação (coordenador E) e entre os profissionais e suas equipes nas unidades básicas de saúde (dentista E2, E3, E1). No município C, alguns identificam essa lacuna e, apesar de conhecerem o planejamento estratégico situacional, assumem a dificuldade de implementá-lo (dentista C3). Outros vêem o planejamento como sinônimo de escala mensal de tarefas (dentista $\mathrm{C} 4, \mathrm{C} 1)$ ou como atividade individual e normativa (dentista C2).

Quanto à dimensão da atenção integral, esse é um problema grave do sistema em ambos os municípios, visto que não há garantia ao paciente de término do tratamento, ou seja, após a primeira consulta, o paciente deve "lutar" por outra vaga, tendo que retornar à fila a cada dia que busque o serviço, situação que funciona como obstáculo à continuidade do cuidado (usuária C4, C2, E1, E3, E4, E7). Essa situação também correspondeu a um acesso difícil e o acolhimento do paciente de má qualidade porque este é obrigado a chegar à unidade cedo, na madrugada, para conseguir nova ficha de atendimento (usuária $\mathrm{C} 4, \mathrm{C} 6$ ) .

Em uma unidade do município E, foi observada uma iniciativa isolada na tentativa de minimizar tal problema por meio da garantia de um percentual de vagas para pacientes em tratamento (dentista E1).

O suporte do nível central para a atenção básica, por intermédio de oficinas de capacitação e supervisão em campo, constituiu-se numa lacuna importante no município E, diferente do município C. Neste, os agentes relatam a realiza- 
Classificação do grau de implantação da atenção à saúde bucal nas dimensões gestão da saúde bucal e práticas assistenciais em dois municípios da Bahia, Brasil, 2003

\begin{tabular}{|c|c|c|c|c|c|}
\hline \multirow[t]{2}{*}{ Dimensões e subdimensões analisadas } & \multirow{2}{*}{$\begin{array}{l}\text { Pontuação máxima } \\
\text { (Proposta) }\end{array}$} & \multicolumn{2}{|c|}{ Município C } & \multicolumn{2}{|c|}{ Município E } \\
\hline & & Pontuação & $\%$ & Pontuação & $\%$ \\
\hline Planejamento e programação & 1,5 & 0,5 & 33,3 * & 0,0 & 0,0 * \\
\hline Diagnóstico epidemiológico & 0,6 & 0,3 & $50,0 * \star$ & 0,0 & 0,0 * \\
\hline Programação & 0,5 & 0,2 & 40,0 ** & 0,0 & 0,0 * \\
\hline Avaliação & 0,4 & 0,0 & 0,0 * & 0,0 & 0,0 * \\
\hline \multicolumn{6}{|l|}{ Suporte da gestão aos profissionais e } \\
\hline às práticas na atenção básica & 0,5 & 0,3 & 60,0 ** & 0,1 & 10,0 * \\
\hline Oferta da assistência odontológica (cobertura potencial) & 0,5 & 0,4 & $80,0 \star \star \star$ & 0,1 & 20,0 * \\
\hline Utilização de serviços (cobertura real) segundo o SIA-SUS & 0,7 & 0,4 & 57,2 ** & 0,15 & 21,4 * \\
\hline Integralidade da atenção em saúde bucal & 1,0 & 0,6 & 60,0 ** & 0,0 & 0,0 * \\
\hline Total gestão da atenção à saúde bucal & 4,2 & 2,2 & 52,4 ** & 0,35 & 8,3 * \\
\hline Deslocamento do eixo sobre os danos para uma ação sobre riscos & 1,0 & 0,5 & 50,0 ** & 0,2 & 20,0 * \\
\hline Práticas educativas desenvolvidas pelos profissionais & 0,9 & 0,6 & $66,7 * \star \star *$ & 0,3 & 33,3 * \\
\hline Atividades junto aos ACS & 0,8 & 0,5 & 62,5 ** & 0,0 & 0,0 * \\
\hline Práticas clínicas & 0,5 & 0,3 & 60,0 ** & 0,1 & 20,0 * \\
\hline Práticas desenvolvidas na família & 0,8 & 0,3 & 25,0 * & 0,0 & 0,0 * \\
\hline Acolhimento e vínculo & 1,0 & 0,3 & 30,0 * & 0,0 & 0,0 * \\
\hline Atenção integral & 0,8 & 0,3 & $37,5^{\star \star}$ & 0,15 & 25,0 * \\
\hline Total de práticas de saúde bucal & 5,8 & 2,8 & $48,3 * \star$ & 0,75 & 12,9 * \\
\hline Grau de implantação (total geral) & 10,0 & 5,0 & 50,0 ** & 1,1 & 11,0 * \\
\hline
\end{tabular}

SIA-SUS: Sistema de Informação Ambulatoriais do SUS; ACS: Agentes Comunitários de Saúde.

* Incipiente;

** Intermediário;

*** Avançado.

ção de oficinas de sensibilização e capacitação dos profissionais, embora com periodicidade variável.

Dentre as práticas de saúde desenvolvidas, aquelas que mais diferenciaram os municípios estudados foram as atividades de treinamento dos ACS para ações de saúde bucal em suas respectivas micro-áreas (dentista C1, C2 e C4) e, curiosamente, as práticas clínicas preventivas desenvolvidas pelos profissionais (dentista C1, C4). De alguma maneira, como prática hegemônica, a atenção individual em consultório tem incorporado, no caso do município C, uma perspectiva mais preventiva (orientação de escovação na cadeira odontológica, maior atenção à condição de higiene oral do paciente) na medida em que complementa o modelo preventivo extra consultório dentário (dentista C1, C4, C3). Outra constatação importante se referiu ao efeito das ações preventivas de base populacional (como grupos educativos na unidade de saúde e os grupos de escovação nas micro-áreas) junto aos usuários. Estes identificam como uma das principais fontes de informação sobre saúde bucal os profissionais da UBS e as atividades por eles desenvolvidas (usuários C2, C7, C8, C4).

Todos os dentistas do município $\mathrm{C}$ realizaram, em alguma medida, treinamento dos ACS para ações básicas de saúde bucal (preventivas) e de diagnóstico para encaminhamento. Contudo, pouca importância tem sido dada à supervisão e ao monitoramento desse trabalho pela análise do cumprimento de metas e da cobertura de ações de saúde bucal nas micro-áreas. Chama atenção o enfoque quase exclusivo da prevenção em saúde bucal para escolares, crianças e, algumas vezes, gestantes.

Chama atenção que os adultos são considerados implicitamente uma geração perdida, uma vez que prevenção e risco são, no discurso dos agentes, quase sempre sinônimo de crianças e doença cárie, respectivamente. Isso se reflete inclusive no discurso dos agentes comunitários de saúde (agentes C3 e C2) porque as atividades de prevenção em saúde bucal em micro-áreas ou em domicílio são voltadas quase exclusivamente para crianças e, às vezes, gestantes e adolescentes. Por outro lado, no município E, não foram 
Figura 2

Classificação do grau de implantação da atenção à saúde bucal segundo o nível da gestão e das práticas de saúde bucal na atenção básica em dois municípios (municípios C e E) da Bahia, Brasil, 2003.

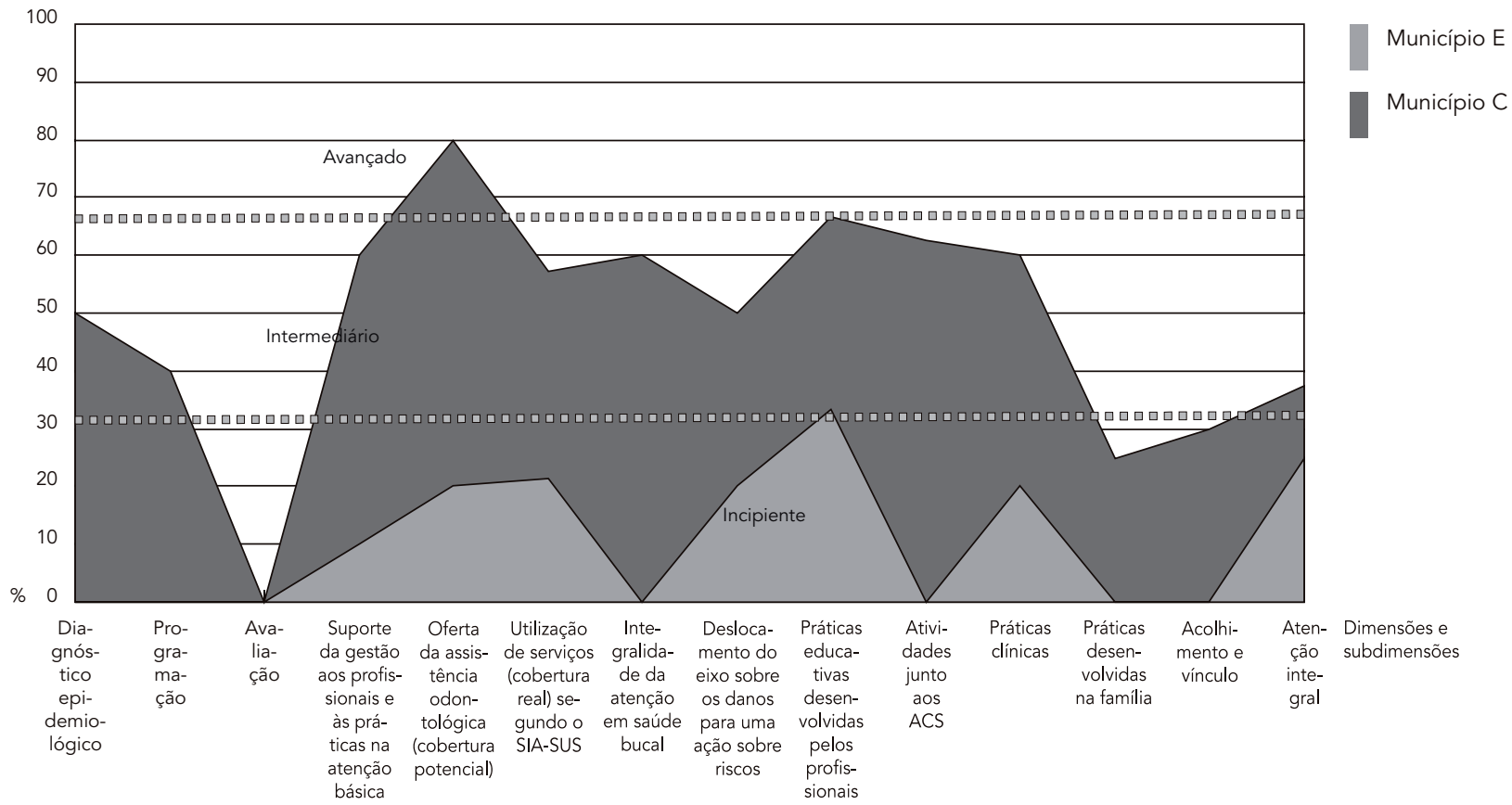

SIA-SUS: Sistema de Informação Ambulatoriais do SUS; ACS: Agentes Comunitários de Saúde.

identificadas ações de controle de riscos conforme a dimensão presente na matriz de implantação (Tabela 2).

O acolhimento foi a dimensão mais crítica identificada em ambos os municípios. A persistência de filas em busca de atendimento e a desumanização pela ausência quase completa de estratégias para acolher as necessidades dos pacientes que procuram a unidade, demonstra ser essa uma questão essencial da prática e que não tem sido respeitada, provocando inquietude e insatisfação entre os profissionais (dentista E2, C3, C2, C1), desestímulo nos pacientes e insegurança sobre seu próprio tratamento (usuários C1, C2, E2, E4). A existência de filas tem sido justificada, em ambos os municípios, por gestores e executores, pelo excesso de demanda. Cabe refletir que, se são áreas com populações adscritas conforme preconiza o modelo de saúde da família, um planejamento estratégico adequado, articulado a um sistema de marcação permanente de consultas, seria capaz de organizar a oferta, sem comprometer a qualidade da atenção, o acolhi- mento e o respeito às necessidades da população da área de abrangência.

O serviço odontológico é considerado o de mais alta demanda nas unidades de saúde e não se verificou em nenhum dos municípios avaliados estratégias de acolhimento adequadas para a humanização do acesso às ações reparadoras de saúde bucal. Vale dizer, o acesso às práticas preventivas em saúde bucal é amplo no município C, mas marcado pelo modelo "sespiano", já que é voltado quase exclusivamente aos escolares e a alguns grupos priorizados, e o acesso às práticas reparadoras é feito por meio da atenção à demanda espontânea, sem organização da oferta por uma programação.

\section{Discussão}

Este estudo revelou que os serviços odontológicos se encontravam organizados predominantemente de forma intermediária, no município C, e incipiente, no município E, quando compara- 
dos com uma "imagem-objetivo" da reorganização das práticas de saúde bucal. O desempenho insuficiente verificado em ambos os municípios estudados pode estar significando que o processo de descentralização não vem sendo acompanhado, no caso da odontologia, de um esforço deliberado, constante e global de melhorar a capacidade administrativa e de gestão dos serviços, apesar do aumento no repasse de recursos financeiros para o setor 9 . Por outro lado, os resultados relativamente melhores apresentados pelo município $\mathrm{C}$ podem estar relacionados com o estágio avançado em que ele se encontrava no que diz respeito à descentralização da gestão global da saúde analisada em estudo anterior 19. Em outras palavras, a existência de um projeto de reorganização das práticas de saúde, de capacidade técnica e governabilidade para implementá-lo constituíram-se em aspectos favoráveis que podem ter influenciado no melhor desempenho observado no que tange às ações específicas de saúde bucal.

Cabe destacar ainda a não-implantação dos componentes relacionados ao planejamento e à programação no município $\mathrm{E}$, e a baixa pontuação obtida nessa dimensão no município C. Chama atenção também a não-implantação de nenhuma ação relacionada à subdimensão "avaliação das estratégias implementadas". Tal fato pode estar relacionado ao pequeno grau de institucionalização dessa prática como ferramenta para a gestão dos serviços de saúde no país 8,19 .

Esses achados são consistentes com aqueles encontrados em outros estudos nos quais a saúde bucal fez parte de um projeto de governo mais amplo como em Campinas 15, e Curitiba 12,15. Apesar disso, ainda que garantidas as condições adequadas no nível local, há um problema de prioridade do setor odontológico dentro das políticas do setor saúde 14. Há um evidente consenso entre os autores acerca da defesa dos princípios e diretrizes orientadores do SUS brasileiro para as políticas de saúde bucal do país 5,6,8,10,12,14,15, apesar de as investigações apontarem para diferentes estratégias de operacionalização dos princípios de universalidade, eqüidade e integra- lidade 10,12,27. Essas diferenças inclusive impõem certa cautela na comparação entre os diferentes modelos de organização das práticas, mesmo na existência de semelhanças entre os governos locais. Há também indicações de que os agentes das práticas (odontólogos) podem desempenhar papel importante quando sustentados por uma razoável gestão e organização dos serviços de saúde bucal, em que se encontram inseridos. A análise dessas características e de seus determinantes em um mesmo contexto de gestão requer a realização de estudos adicionais.

A presente investigação confirmou o que análises anteriores apontavam acerca dos potenciais da descentralização. Isto é, que o referido processo não é uma panacéia como corretamente analisaram alguns autores no início da década de noventa 28,29 , não sendo capaz de gerar automaticamente mudanças nos serviços de saúde. Além disso, também revelou que o financiamento por si só não é suficiente - diante do mesmo volume de recursos, houve diferentes impactos na organização do setor de saúde bucal nos dois municípios avaliados.

Para assegurar comparabilidade na avaliação e no monitoramento da descentralização da atenção à saúde bucal é necessário levar em conta ainda diversas características dos modelos de atenção. A “imagem-objetivo” aqui formulada pode se constituir em ponto de partida para o desenvolvimento e aperfeiçoamento de instrumentos capazes de subsidiar esse processo. A matriz elaborada com essa finalidade pode ser redimensionada em estudos posteriores mediante inclusão de novas dimensões e critérios considerando as especificidades das regiões do país. A sua submissão a um comitê de experts correspondeu a uma validação preliminar. A comparação entre estudos de caso contribuiu para aumentar sua validade externa que poderá ser ampliada com a sua utilização em escala maior quando então poderá ser submetida ao consenso de outros atores sociais. As informações disponibilizadas pelo instrumento poderão subsidiar os gestores no aperfeiçoamento das suas diferentes estratégias de reorganização das práticas de saúde bucal, visando ao controle dos principais problemas desta área. 


\section{Resumo}

Com o objetivo de discutir as relações entre a descentralização da gestão da saúde e as características da atenção à saúde bucal foi realizado um estudo comparado de dois casos exemplares em municípios selecionados do Estado da Bahia, Brasil, em diferentes estágios de descentralização da gestão da saúde. As informações foram obtidas por intermédio de entrevistas semi-estruturadas com informantes-chave, observação das práticas e análise de dados secundários do Sistema de Informação Ambulatorial (SIA-SUS). Os resultados são indicativos da existência de influência de características do governo municipal e da gestão da saúde para a organização da atenção à saúde bucal. Essa influência, contudo, foi inferior ao esperado. O município $C$, classificado como em estágio avançado da descentralização, apresentou padrão intermediário de implantação das ações de saúde bucal (50\%), enquanto o município E, em estágio incipiente em relação à descentralização também se apresentou incipiente no que se refere aos serviços públicos odontológicos, com apenas $11 \%$ de implantação. Esse estudo revelou que a descentralização não tem sido acompanhada de melhorias na capacidade de gestão dos serviços odontológicos, apesar do aumento no repasse de recursos financeiros para esta área.

Saúde Bucal; Avaliação em Saúde; Política de Saúde

\section{Colaboradores}

S. C. L. Chaves elaborou a pergunta de investigação, coletou os dados e construiu teoricamente o projeto de investigação. L. M. Vieira-da-Silva orientou a condução dos trabalhos, contribuiu na construção teórico-metodológica da investigação e revisou o texto.

\section{Referências}

1. Ministério da Saúde. Relatório da XI Conferência Nacional de Saúde. Brasília: Ministério da Saúde; 2000.

2. Viana ALd'A, Heimann LS, Lima LD, Oliveira RG, Rodrigues SH. Mudanças significativas no processo de descentralização do sistema de saúde no Brasil. Cad Saúde Pública 2002; 18 Suppl:S139-51.

3. Teixeira CF, Paim JS, Villasboas AL. SUS, modelos assistenciais e vigilância da saúde. In: Paim JS, organizador. Promoção e vigilância da saúde. Salvador: Editora CEPS; 2002. p. 23-57.

4. Ministério da Saúde. Portaria no. 95/MS. Norma Operacional da Assistência à Saúde (NOAS). Brasília: Ministério da Saúde; 2001.

5. Cordón J. A construção de uma agenda para a saúde bucal coletiva. Cad Saúde Pública 1997; 13:557-63.
6. Souza DS, Cury JA, Caminha JN, Ferreira MA, Tomita NE, Narvai PC, et al. A inserção da saúde bucal no Programa Saúde da Família. Revista Brasileira de Odontologia em Saúde Coletiva 2001; 2:7-29.

7. Ministério da Saúde. Saúde bucal na atenção básica: texto inicial formulado pela Coordenação Nacional de Saúde Bucal para debate no fórum de coordenadores estaduais. Brasília: Ministério da Saúde; 2003.

8. Zanetti CHG. Saúde Bucal no Programa Saúde da Família (PSF) - proposição e programação. http://www.saudebucalcoletiva.unb.br (acessado em 08/Fev/2001).

9. Ministério da Saúde. Investimento em saúde bucal passa de 84 milhões para 238 milhões em 2004. http://www.saude.gov.br (acessado em 02/ Jun/2004). 
10. Silveira Filho AD. A saúde bucal no PSF: o desafio de mudar a prática. Brasília: Divisão Nacional de Saúde Bucal, Ministério da Saúde; 2003.

11. Ministério da Saúde. Portaria no. 1.121/MS. Pacto da atenção básica 2002. Brasília: Ministério da Saúde; 2002.

12. Roncalli AGO. A organização da demanda em serviços públicos de saúde bucal: universalidade, eqüidade e integralidade em saúde bucal coletiva [Tese de Doutorado]. Araçatuba: Faculdade de Odontologia, Universidade Estadual Paulista Júlio de Mesquita Filho; 2000.

13. Peres MAA. A saúde bucal em um sistema local de saúde: estudo de caso no município de Ipatinga, MG, Brasil 1989-1994 [Dissertação de Mestrado]. São Paulo: Faculdade de Saúde Pública, Universidade de São Paulo; 1995.

14. Werneck MAF. A saúde bucal no SUS: uma perspectiva de mudança [Tese de Doutorado]. Niterói: Universidade Federal Fluminense; 1994.

15. Serra CG. A saúde bucal como política de saúde. Análise de três experiências recentes: Niterói, Campinas e Curitiba [Dissertação de Mestrado]. Rio de Janeiro: Instituto de Medicina Social, Universidade do Estado do Rio de Janeiro; 1998.

16. Chaves SCL, Vieira-da-Silva LM. As práticas preventivas no controle da cárie dental: uma síntese de pesquisas. Cad Saúde Pública 2002; 18:129-39.

17. Denis J, Champagne F. Análise da implantação de programas. In: Hartz ZMA, organizador. Avaliação em saúde: dos modelos conceituais à prática na análise da implantação de programas. Rio de Janeiro: Editora Fiocruz; 1997. p. 49-88.

18. Ministério da Saúde. Norma Operacional Básica do Sistema Único de Saúde/NOB-SUS96. Gestão plena com responsabilidade pela saúde do cidadão. Brasília: Ministério da Saúde; 2002.

19. Vieira-da-Silva LM, Hartz ZMA, Chaves SCL, Silva GAP, Paim JS. Análise da implantação da gestão descentralizada em saúde: estudo comparado de cinco casos na Bahia, Brasil. Cad Saúde Pública 2007; 23:355-7.

20. Rossi PH, Freeman HE. Strategies for impact assessment. In: Rossei PH, Freeman HE, editrs. Evaluation: a sistematic approach. Newbury Park: Sage Publications; 2003. p. 225-69.
21. Matus C. Política, planificación y gobierno. Caracas: Fundación Altadir; 1987.

22. Donabedian A. Methods of deriving the criteria. In: Donabedian A, editor. Explorations in quality assessment and monitoring. Ann Arbor: Health Administration Press; 1982. p. 504-70.

23. Jones J, Hunter D. Using the Delphi and nominal group technique in health services research. In: Pope C, Mays N, editors. Qualitative research in health care. London: BMJ Books; 2000. p. 40-9.

24. Ministério da Saúde. Portaria n. 1.101/GM. Aprova os principais padrões de produção ambulatorial dos serviços de saúde do SUS. Brasília: Ministério da Saúde; 2002.

25. Departamento de Informação e Informática do SUS. Informações de saúde. http://www.datasus. gov.br (acessado em Jan/2004).

26. Gomes R. A análise dos dados em pesquisa qualitativa. In: Gomes R, organizador. Pesquisa social: teoria, método e criatividade. Petrópolis: Editora Vozes; 2001. p. 67-81.

27. Cordón J. A inserção da odontologia no SUS: avanços e dificuldades [Tese de Doutorado]. Brasília: Universidade de Brasília; 1998.

28. Matus C. La descentralización: una nueva panacea para impulsar el desarrollo local? Cuadernos del CLAEH 1989; 51:57-75.

29. Paim JS. Quando a municipalização não é o caminho. Tema (Proj RADIS) 1991; 12:27-8.

30. Instituto Brasileiro de Geografia e Estatística. Dados demográficos, sociais e sanitários. http://www. ibge.gov.br/home (acessado em Jul/2004).

31. Departamento de Informação e Informática do SUS. Informações de saúde. http://w3.datasus.gov. br/datasus/datasus.php (acessado em Jul/2004).

32. Departamento de Informação e Informática do SUS. Informações financeiras de transferências a municípios. http://portal.saude.gov.br/portal/ saude/Gestor/area.cfm?id_area=347 (acessado em Jul/2004).

33. Programa das Nações Unidades para o Desenvolvimento. Atlas de desenvolvimento humano no Brasil (IDH) http://www.pnud.org.br/atlas/oque/ index.php (acessado em Ago/2004).

Recebido em 20/Dez/2005

Versão final reapresentada em 13/Jun/2006

Aprovado em 09/Nov/2006 